\title{
Generalized tolerance sensitivity and DEA metric sensitivity
}

\author{
Luka Neralici $^{1, *}$ and Richard E. Wendell ${ }^{2}$ \\ ${ }^{1}$ Faculty of Economics and Business, University of Zagreb \\ Trg J. F. Kennedy 6, 10000 Zagreb, Croatia \\ E-mail: 〈lneralic@efzg.hr〉 \\ ${ }^{2}$ Katz Graduate School of Business, University of Pittsburgh \\ Pittsburgh, PA 15260, USA \\ E-mail: 〈wendell@pitt.edu〉
}

\begin{abstract}
This paper considers the relationship between Tolerance sensitivity analysis in optimization and metric sensitivity analysis in Data Envelopment Analysis (DEA). Herein, we extend the results on the generalized Tolerance framework proposed by Wendell and Chen and show how this framework includes DEA metric sensitivity as a special case. Further, we note how recent results in Tolerance sensitivity suggest some possible extensions of the results in DEA metric sensitivity.
\end{abstract}

Keywords: sensitivity, tolerance, DEA, metric, stability, Chebyshev

Received: September 22, 2014; accepted: March 3, 2015; available online: March 30, 2015

DOI: $10.17535 /$ crorr.2015.0014

\section{Introduction}

Tolerance sensitivity analysis in linear programming was proposed by Wendell $[19,20,21]$. This work was originally focused on the Chebyshev norm and showed how to find a maximum tolerance, typically interpreted as the maximum percentage within which selected coefficients or terms (e.g., objective function coefficients) could vary while maintaining the same optimal basis. As noted by Ward and Wendell [18], Wendell [22], and Wendell and Chen [24], a number of subsequent papers proposed various theoretical extensions as well as applications including sensitivity of matrix coefficients, facility location problems, and multiple objective problems.

Shortly before Tolerance sensitivity was proposed, Charnes et al. [2] wrote the seminal paper on Data Envelopment Analysis (DEA), proposing a methodology for performing a relative efficiency evaluation of entities called Decicsion Making Units (DMUs) which use the same inputs and produce the

${ }^{*}$ Corresponding author 
same outputs. Since then, DEA has become a fast growing area with, according to the bibliography of Emrouznejad [9], more than four thousand references of works on DEA. For an excellent introduction to the theory and use of DEA see, for example, [7] and [6]. The importance of variations in the data, or what we will call sensitivity analysis, was apparent in the early days beginning with Charnes et al [1] considering sensitivity with respect to a single output. This was followed by Charnes and Neralić [4], Neralić [13], Neralić and Wendell [14] and others. One of the approaches to DEA sensitivity, proposed by Charnes et al $[3,5]$, was to apply metric concepts in a way to make it possible to determine allowable variations in all inputs and outputs for one DMU in a DEA model. In particular, using an $l_{p}$ norm to characterize distance, these papers propose optimization models to determine a maximum radius, called a "radius of stability", within which variations of the inputs and outputs in a selected DMU would remain efficient, if the selected DMU was originally efficient, and would remain inefficient, if it was originally inefficient. Since then, research on DEA metric sensitivity has primarily focused on the Chebyshev norm. For a further discussion see, for example, [8] as well as [7] and [6].

Both Tolerance sensitivity and DEA metric sensitivity have remarkable similarities, but interesting differences. Herein, we extend the results on a generalized Tolerance framework proposed in [24] and we show how the generalized framework includes DEA metric sensitivity as a special case. Further we note how recent results in Tolerance sensitivity suggest extensions of the results for DEA metric sensitivity.

The paper proceeds as follows. Section 2 gives a statement of the general Tolerance framework from Wendell and Chen together with some theoretical extensions. Section 3 applies this general framework to DEA, yielding the results of Charnes et al [3], Cooper et al [8], and Cooper et al [7] on metric-based DEA sensitivity analysis. Section 4 gives some concluding observations and discusses potential future research, including how recent results in Tolerance sensitivity can be applied to yield extensions of the results in DEA metric sensitivity.

\section{A generalized tolerance sensitivity analysis framework ${ }^{1}$}

Let $c$ denote a vector of parameters in $R^{t}$, and let $f(x, c)$ and $g(x, c)$ denote vectors of functions on $X$ with parameters $c$ where $X \subseteq R^{n}$ is a given constraint set. Consider the optimization problem:

$$
\operatorname{Max}\{f(x, c): g(x, c) \leq 0, x \in X\}
$$

\footnotetext{
${ }^{1}$ In this paper we follow and extend the notation given in Wendell and Chen [24]. Any overlap between this notation and notation used in DEA will be clear from the context.
} 
Observe that when the vector $f$ has multiple components, (1) denotes a multiple objective problem. Let $x^{*}$ denote a feasible solution to (1) when $\mathrm{c}=\hat{c}$.

Consider a perturbation structure of $c$ in which $\mathrm{c}=\hat{c}+\curlyvee C^{\prime}$ where $C^{\prime}$ is a given $s \times t$ matrix and where the parameters $\gamma$ can take on values in a given set $\Gamma$, called an a priori parametric region, denoting a region within which $\gamma$ may vary. The a priori set $\Gamma$, which always includes 0 and which we assume is closed, enables us to exploit known a priori limits on variations of the parameters $\gamma$ to obtain stronger results. Herein we consider the case when $s=t$ and $C^{\prime}$ is a nonnegative, diagonal matrix. The diagonal elements $C^{\prime}{ }_{i i}$ are sometimes called perturbation rates and denoted as a vector $c^{\prime}$. Observe that when $\gamma=0$ we have $c=\hat{c}$.

Suppose that when $\gamma=0$ the solution $x^{*}$ satisfies some specified optimality condition, often characterized as a set of equations and/or inequalities in $\gamma$. For example, in a linear programming problem the optimality condition could be that the same basis is optimal, that the same solution is optimal, that the same variables are basic, etc. Let $P\left(x^{*}\right)$, called a critical region, denote the set of $\curlyvee$ for which the specified optimality condition holds. For problems with a linear structure, $P\left(x^{*}\right)$ is typically a convex polyhedron. A basic question in sensitivity analysis is: How much can $\gamma$ deviate from 0 within $\Gamma$ and still remain in the critical region $P\left(x^{*}\right)$ ? To eliminate the trivial case, we assume that $P\left(x^{*}\right)$ is a proper subset of $R^{s}$. Tolerance sensitivity analysis is an approach to answer this question in the case when "how much" is determined by the size of a hyperbox within which the parameters $\gamma$ can vary simultaneously and independently over $\Gamma$. For a further discussion see, for example, [18, 22, 24, 11].

The Tolerance sensitivity problem can be characterized as:

$$
\begin{array}{ll} 
& \sup \rho(u) \\
\text { s.t. } & \gamma \in \Gamma \cap\{\gamma: \gamma \in B(u)\} \Rightarrow \gamma \in P\left(x^{*}\right)
\end{array}
$$

where $u$ is a nonnegative vector in $R^{2 t}$,

$B(u)$ is a hyperbox, also called a tolerance box, characterized as

$B(u)=\left\{\gamma:-u_{i} \leq \gamma_{i} \leq u_{t+i}, \quad\right.$ for $\left.i=1,2, \cdots, t\right\}$,

$\rho(u)$ denotes a function that characterizes the size of the hyperbox

$" \Longrightarrow$ " denotes an "if-then" implication.

If $u$ denotes a feasible solution to (2), observe that the parameters $\checkmark$ may vary simultaneously and independently over the region $\mathrm{B}(u) \cap \Gamma$ while preserving the specified optimality condition for $x^{*}$.

Traditional Tolerance sensitivity can be viewed as setting all components of $u$ equal to one number, which we call a tolerance and denote as $\tau$. In this case, the hyperbox is a hypercube corresponding to the set $\left\{\gamma:\|\gamma\|_{\infty} \leq \tau\right\}$, where \|\|$_{\infty}$ is 
the Chebyshev norm and the tolerance $\tau$ denotes the size $\rho(u)$ of the hypercube. Problem (2) corresponds to finding the maximum tolerance $\tau$, denoted as $\tau^{*}$, where the optimality condition $\gamma \in P\left(x^{*}\right)$ holds for all $\gamma \in \Gamma$ when $\|\gamma\|_{\infty} \leq \tau$. Thus, the traditional Tolerance problem for computing $\tau *$ can be stated as:

$$
\begin{array}{ll} 
& \sup \tau \\
\text { s.t. } & \gamma \in \Gamma \cap\left\{\gamma:\|\gamma\|_{\infty} \leq \tau\right\} \Rightarrow \gamma \in P\left(x^{*}\right)
\end{array}
$$

Observe that the maximum tolerance $\tau *$ is itself a tolerance iff the sup in (3) is achieved. Also note that the maximum tolerance corresponds to a hypercube $\mathrm{B}(\bar{u})$ where $\bar{u}_{i}=\tau^{*}$ for all $\mathrm{i}$. When $\mathrm{c}^{\prime}=\hat{c}$ then $\tau^{*} \mathrm{x} 100 \%$, called the maximum Tolerance percentage, denotes the maximum percentage within which the parameters $c$ may vary simultaneously and independently from their original values $\hat{c}$ while maintaining the optimality property. In addition, observe that setting a component $c_{i}{ }^{\prime}=0$ is a convenient way to specify that no perturbations on $\hat{C}_{i}$ will be considered, which would be the case if we knew that $\hat{C}_{i}$ was the known value of $c_{i}$. A key advantage of using traditional Tolerance sensitivity is the ease with which a manager can intuitively interpret and readily use the maximum tolerance percentage in sensitivity analysis. For example, in considering sensitivity analysis of the objective function coefficients in linear programming, the maximum tolerance percentage can denote the maximum percentage by which the objective function coefficients may vary from their original values while maintaining the same optimal basic feasible solution.

From (3) we can give a simple characterization of the case when $\tau^{*}=\infty$.

Theorem 1. The maximum tolerance $\tau^{*}=\infty$ iff

$$
\gamma \in \Gamma \Rightarrow \gamma \in P\left(x^{*}\right)
$$

Observe that, for the special case wnen tnere is no a priorı intormation on the allowable range of perturbations (namely, when $\left.\Gamma=R^{t}\right), \tau^{*}$ is finite iff $\mathrm{P}\left(\mathrm{x}^{*}\right)$ is a proper subset of $\mathrm{R}^{\mathrm{t}}$. Letting $\mathrm{P}\left(\mathrm{x}^{*}\right)^{\mathrm{c}}$ denote the relative complement of $\mathrm{P}\left(\mathrm{x}^{*}\right)$, we now give an equivalent characterization of (3).

Theorem 2. An equivalent representation of (3) is

$$
\begin{array}{ll} 
& \inf \|\gamma\|_{\infty} \\
\text { s.t. } & \gamma \in\left\{\Gamma \cap P\left(x^{*}\right)^{c}\right\}
\end{array}
$$

Proof. The equivalence when $\tau^{*}=\infty$ follows from Theorem 1. Thus, consider the case when $\tau^{*}$ is finite, letting inf denote the infimum in (5). We first show that $\tau^{*} \leq$ inf. If $\tau^{*}>$ inf, then by (5) there exists a $\gamma^{\prime} \in\left\{\Gamma \cap P\left(x^{*}\right)^{c}\right\}$ with 
$\left\|\gamma^{\prime}\right\|_{\infty}<\tau^{*}$. But letting $\tau=\left\|\gamma^{\prime}\right\|_{\infty}$ in (3) gives a contradiction. We now show that inf $\leq \tau^{*}$. Consider a positive sequence $\left\{\varepsilon^{k}\right\}$ converging to 0 . For each $k$, let $\tau^{k}=\tau^{*}+\varepsilon^{k}$. For each $k$ there is by (3) some $\gamma^{k} \in \Gamma$ with $\left\|\gamma^{k}\right\|_{\infty} \leq \tau^{k}$ and $\gamma^{k} \in P\left(x^{*}\right)^{c}$. Since for each $k$, inf $\leq\left\|\gamma^{k}\right\|_{\infty} \leq \tau^{k}$, it follows that inf $\leq \tau^{*}$. QED

The set $P\left(x^{*}\right)^{c}$ in $(5)$ is often not closed. In the following important special case, when there is no a priori information on the variations of the parameters, this presents no problem.

Theorem 3. If $\Gamma=R^{t}$ then $\tau^{*}$ equals the infimum

$$
\begin{array}{cc} 
& i n f \\
\text { s.t. } & \gamma \in \overline{P\left(x^{*}\right)^{c}}
\end{array}
$$

The proof is immediate. Note that, since problems (3) and (6) are equivalent when $\Gamma=\mathrm{R}^{\mathrm{t}}$, one can choose whichever problem is easier to solve for a particular situation. Often (e.g., for Tolerance sensitivity in linear programming) problem (6) is used. However in DEA, as we will see, problem (3) is easiest when evaluating the Tolerance sensitivity of an inefficient DMU.

When $\Gamma$ does not equal $R^{t}$ dealing with the closure of $P\left(x^{*}\right)^{c}$ is more delicate. This is illustrated in the example below where the infimum in (5) is infinite but where substituting the closure of $P\left(x^{*}\right)^{c}$ for $P\left(x^{*}\right)^{c}$ in (5) gives a finite infimum.

Example. Consider the simple linear programming problem: Max $\left\{\left(2 x_{1}+1 x_{2}\right)\right.$ : $\left.x_{1}+x_{2}=1, x_{1}, x_{2} \geq 0\right\}$ for which $x^{*}=(1,0)$. Letting $c^{\prime}=(1,1)$, the perturbed problem is

$\operatorname{Max}\left\{\left(2+\gamma_{1}\right) x_{1}+\left(1+\gamma_{2}\right) x_{2}: x_{1}+x_{2}=1, x_{1}, x_{2} \geq 0\right\}$. Thus, we have $P\left(x^{*}\right)=$ $\left\{\left(\gamma_{1}, \gamma_{2}\right): \gamma_{2}-\gamma_{1} \leq 1\right\}$. If we have the a priori information that $\Gamma=\left\{\left(\gamma_{1}, \gamma_{2}\right)\right.$ : $\left.\gamma_{1} \geq-.5, \gamma_{2} \leq .5\right\}$, then the infimum in (5) is infinity but the infimum in (5) with the closure of $P\left(x^{*}\right)^{c}$ substituted for $P\left(x^{*}\right)^{c}$ is .5 .

Building on the work of Wendell [20] and others, the solution of (5) when $\Gamma$ does not equal $R^{t}$ is explored in a subsequent paper.

Typically, the critical region $P\left(x^{*}\right)$ in applications of Tolerance sensitivity corresponds to a convex polyhedron. In this case, as noted by Hladik [11], the basic idea of Tolerance sensitivity in linear systems as given by (2) is to find "a maximal box lying inside a convex polyhedron". Here a box $B(u)$ being called "maximal" means that there is no other box lying in $P\left(x^{*}\right)$ and being a strict superset of $B(u)$. Note that the box given by traditional Tolerance sensitivity is 
maximal among the set of boxes that are hypercubes. Further, the work on Tolerance sensitivity has focused on the case when $P\left(x^{*}\right)$ has an external representation $^{2}$ such as $P\left(x^{*}\right)=\{\gamma: D \gamma \leq d\}$. As we will see, DEA metric sensitivity is one application for which the tolerance framework using an internal representation of a polyhedron is used.

\section{DEA metric sensitivity as Tolerance sensitivity}

We now consider an application to DEA. As usual in DEA, we assume that there are $n$ DMUs to be evaluated with $n>1$, where each consumes given amounts of $m$ different inputs to produce given amounts of $s$ different outputs. Specifically, let $X_{j} \in R^{m}$ denote the vector of inputs used by $\mathrm{DMU}_{j}$ and let $Y_{j} \in$ $R^{s}$ denote the vector of corresponding outputs. We assume that $X_{j} \geq 0$ and $Y_{j} \geq$ 0 . Let $X_{i j}$ and $Y_{r j}$ denote components $i$ and $r$ of the respective vectors and, as common in DEA, assume that each DMU has at least one positive input and one positive output component. Following [3] and [7, 8], we focus for simplicity on the Additive Model which has the production possibility set $S=\{(x, y): x \geq$ $\left.\sum_{j=1}^{n} \lambda_{j} X_{j}, y \leq \sum_{j=1}^{n} \lambda_{j} Y_{j}, \sum_{j=1}^{n} \lambda_{j}=1, \lambda \geq 0\right\}$. For any two given points $\left(x^{\prime}, y^{\prime}\right)$ and $\left(x^{\prime \prime}, y^{\prime \prime}\right)$ in $S$ we say that $\left(x^{\prime}, y^{\prime}\right)$ dominates $\left(x^{\prime \prime}, y^{\prime \prime}\right)$ if $x^{\prime} \leq x^{\prime \prime}$ and $y^{\prime} \geq y^{\prime \prime}$ with at least one inequality being strict. A given point $\left(x^{*}, y^{*}\right) \in S$ is said to be efficient if there is no point $(x, y) \in S$ that dominates it. Otherwise it is said to be inefficient. As shown by Charnes et al [3], testing whether or not a point ( $x$, $y$ ) is efficient can be easily determined by solving an auxiliary linear program.

Let $\mathrm{DMU}_{0}$ denote a selected $\mathrm{DMU}$ with corresponding inputs and outputs $X_{0}$ and $Y_{0}$. As in [3] we consider a radius of stability with respect to a given norm within which the inputs and outputs of $\mathrm{DMU}_{0}$ may vary while preserving a DMU's current classification - efficient or inefficient. Following [3] and [7, 8], we assume that the input and output values of all other DMUs remain unchanged, and we focus on the Chebyshev $\left(l_{\infty}\right)$ norm. In particular, for the Chebyshev norm observe that the radius of stability is a number $\tau^{*} \geq 0$ satisfying the following property where $d_{r}^{+}$and $d_{i}^{-}$are positive numbers:

$$
0 \leq \tau<\tau^{*}, x_{i} \in\left[X_{i 0}-\tau d_{i}^{-}, X_{i 0}+\tau d_{i}^{-}\right], y_{r} \in\left[Y_{r 0}-\tau d_{r}^{+}, Y_{r 0}+\tau d_{r}^{+}\right],
$$

and $\mathrm{DMU}_{0}$ is efficient (inefficient) implies that $(x, y)$ is also efficient (inefficient); and $\tau *$ is the largest number for which this property holds.

\footnotetext{
${ }^{2}$ For a discussion of an external and internal representations of a polyhedron see, for example, page 107 of Schrijver [15].
} 
Charnes et al [3] and Cooper et al [7, 8] give the following results.

Theorem 4. If $D M U_{0}$ is inefficient then

$$
\begin{array}{ll} 
& \tau^{*}=\operatorname{Max} \theta \\
\text { s.t. } & \sum_{j=1}^{n} Y_{r j} \lambda_{j}-\theta d_{r}^{+} \geq Y_{r 0} \text { for } r=1, \ldots, s \\
& \sum_{j=1}^{n} X_{i j} \lambda_{j}+\theta d_{i}^{-} \leq X_{i 0} \text { for } i=1, \ldots, m \\
& \sum_{j=1}^{n} \lambda_{j}=1
\end{array}
$$

$\lambda, \theta \geq 0$

Theorem 5. If $D M U_{0}$ is efficient then

$$
\begin{array}{ll} 
& \tau^{*}=\text { Min } \theta \\
\text { s.t. } & \sum_{j=1, j \neq 0}^{n} Y_{r j} \lambda_{j}+\theta d_{r}^{+} \geq Y_{r 0} \text { for } r=1, \ldots, s \\
& \sum_{j=1, j \neq 0}^{n} X_{i j} \lambda_{j}-\theta d_{i}^{-} \leq X_{i 0} \text { for } i=1, \ldots, m \\
\sum_{j=1, j \neq 0}^{n} \lambda_{j}=1 \\
\lambda, \theta \geq 0
\end{array}
$$

where $j \neq 0$ refers to the efficient $D M U_{0}$ that is being analyzed. In particular, note that if all components of $d^{-}$and $d^{+}$equal 1 , then Theorems 4 and 5 above respectively correspond to Theorems 3.4 and 3.2 in [3].

The following multiple optimization problem for the Additive DEA model corresponds to problem (1) in Tolerance sensitivity:

$$
\begin{array}{ll} 
& \operatorname{Min} x \\
& \operatorname{Max} y \\
\text { s.t. } & (x, y) \in S
\end{array}
$$

Let the coordinates $X_{0}$ and $Y_{0}$ of $\mathrm{DMU}_{0}$ correspond to the feasible solution $x^{*}$ in (1). With $\left(X_{0}, Y_{0}\right)$ denoting an efficient (inefficient) DMU, we now show how Theorems 4 and 5 above can be viewed as an application of Tolerance sensitivity to problem (10). 
Letting $C^{\prime}=\left(C^{\prime}{ }_{X}, C^{\prime}{ }_{Y}\right)$ whose nonnegative diagonal elements are $d_{i}^{-}$and $d_{r}^{+}$, we can represent points $x$ and $y$ resulting from perturbations from $X_{0}$ and $Y_{0}$ as $\left(X_{0}+\gamma_{X} C^{\prime}{ }_{X}\right)$ and $\left(Y_{0}+\gamma_{Y} C^{\prime}{ }_{Y}\right)$ respectively. Observe that if $d^{-}=X_{0}$ and $d^{+}=Y_{0}$ then $\gamma_{X}$ and $\gamma_{Y}$ represent $\%$ variations of $x$ and $y$ from $X_{0}$ and $Y_{0}$ respectively as in (7). This corresponds to an observation in [8] that $\tau^{*}$ in Theorems 4 and 5 can be interpreted as a ratio relative to the positive weights used for each constraint. Also, in a similar analysis for the Ratio (CCR) model, Charnes et al [5] observe that $\tau$ * denotes "the minimum percentage change necessary in every input and output to bring the test unit to its nearest unstable point". Note that the interpretations of the perturbations are identical to those in Tolerance sensitivity.

Below we individually consider the cases when $\mathrm{DMU}_{0}$ is inefficient and efficient.

\subsection{When $\mathrm{DMU}_{0}$ is inefficient}

First we assume that $\mathrm{DMU}_{0}$ is inefficient. In this case the critical region $P\left(x^{*}\right)$ is the set for which a perturbation gives an inefficient DMU. Thus, when $\mathrm{DMU}_{0}$ is inefficient, note that $P\left(x^{*}\right)$ equals the set of perturbations $\left(\gamma_{X}, Y_{Y}\right)$ where $\left(X_{0}\right.$ $\left.+\gamma_{X} C^{\prime \prime}{ }_{X}, Y_{0}+\gamma_{Y} C^{\prime}{ }_{Y}\right)$ is in the convex hull of $\left\{\left(X_{j}, Y_{j}\right): j=1, \ldots, n\right\}$ plus the polyhedral cone, excluding the point 0 , generated by $\left\{\left(e_{i}, 0\right): i=1, \ldots, m\right\}$ and $\left\{\left(0,-e_{r}\right): r=1, \ldots, s\right\}$.

Consider the traditional Tolerance problem (3). Observe that problem (3) is equivalent to problem (8) when $\Gamma$ has no limits. Since the supremum in (3) is identical whether or not we exclude 0 from the polyhedral cone, we can include the 0 point in the cone, so that $P\left(x^{*}\right)$ corresponds to an interior representation of a polyhedron. Interestingly, in contrast to Wendell [21] and Hladik [11] who give a closed form solution for an external representation of a polyhedron $P\left(x^{*}\right)$ when $\Gamma=R^{t}$ (based on computing the minimum distances to each face of the polyhedron), Charnes et al [3] and Cooper et al [7,8] deal directly with an internal representation that exploits the structure of $\mathrm{P}\left(\mathrm{x}^{*}\right)$ by showing that an optimal solution to (3) can be found by moving from $\left(X_{0}, Y_{0}\right)$ in the direction $\theta\left(-d_{i}^{-}, d_{r}^{+}\right)$. In doing this, they obtain (8) in Theorem 4 as an equivalent optimization model to the traditional Tolerance problem (3). Thus, for an inefficient DMU we conclude that DEA metric sensitivity is a special case of the generalized tolerance framework. 


\subsection{When $\mathrm{DMU}_{0}$ is efficient}

Now suppose that $\mathrm{DMU}_{0}$ is efficient. In this case, the critical region $P\left(x^{*}\right)$ is the set for which a perturbation corresponds to an efficient DMU; and therefore $P\left(x^{*}\right)^{c}$ corresponds to inefficient DMUs. Thus, $P\left(x^{*}\right)^{c}$ equals the set of perturbations $\left(\gamma_{X}, \gamma_{Y}\right)$ where $\left(X_{0}+\gamma_{X} C^{\prime}{ }_{X}, Y_{0}+\gamma_{Y} C^{\prime}{ }_{Y}\right)$ is in the convex hull of $\{(X j, Y j): j=1, \ldots, n\}$ plus the polyhedral cone, excluding the point 0 , generated by $\left\{\left(e_{i}, 0\right): i=1, \ldots, m\right\}$ and $\left\{\left(0,-e_{r}\right): r=1, \ldots, s\right\}$. In particular, observe that $P\left(x^{*}\right)$ is a proper subset of $R^{m+s}$.

Since the case above corresponds to having $\Gamma=R^{t}$, it follows from Theorem 3 that (6) is an equivalent characterization of (3). Charnes et al [3] and Cooper et al $[7,8]$ essentially observe that problem (6) is the linear programming problem (9) given in Theorem 5. Thus, for an efficient DMU we conclude again that DEA metric sensitivity is a special case of the generalized tolerance framework.

\section{Conclusions and observations}

This paper considers the relationship between Tolerance sensitivity analysis in optimization and metric sensitivity analysis in DEA. Specifically, it extends the results on the generalized Tolerance framework proposed by Wendell and Chen and shows how this framework includes DEA metric sensitivity as a special case. Further, as noted below, the paper shows how recent results in Tolerance sensitivity suggest some possible extensions of the results in DEA metric sensitivity.

In a related paper Singh [16] recently proposed a volume-maximizing tolerance approach for DEA metric sensitivity. This approach, while not directly related to the results of Charnes et al [3] and Cooper et al [7, 8], offers an alternative method of constructing a stability region by maximizing the volume of the region (building on the work of Wang and Huang [17]). Critiques of the volume-maximizing approach include: a lack of an intuitive rationale for the maximizing volume objective (see [22]); and the need to solve a nonlinear optimization problem to perform sensitivity analysis on an optimal basis of a linear program (see [10]). Also, while not directly relevant herein, we observe that Jahanshahloo et al [12] has recently shown how models proposed by Charnes et al [3] and Cooper et al [7, 8] can be combined into one model with the same results, thereby saving some computational work.

One conclusion from the results herein is that DEA metric sensitivity uses a similar framework to traditional Tolerance sensitivity. However, the relationship between them is only clear when considering the general framework given herein. Furthermore, there are significant differences in DEA metric sensitivity from previous work in Tolerance sensitivity. One is that in contrast 
to considering sensitivity with respect to just optimal solutions, DEA metric sensitivity considers variations of an inefficient (i.e., a "nonoptimal") solution. Another is that DEA considers both internal and external representations of a polyhedron, whereas traditional Tolerance sensitivity considers just external representations.

Given the relationship between DEA metric sensitivity and Tolerance sensitivity, it is interesting to consider how recent work in Tolerance sensitivity can yield extensions of DEA metric sensitivity results. In particular, building on the results of Filippi [10], Hladik [11], and Wendell [20], a follow-up paper by the authors herein show how to obtain larger maximum tolerances by exploiting a priori parametric information and how to expand the maximum tolerance region. Interestingly, the DEA - Tolerance relationship also suggests other possible research topics such as building on the work of Wendell [23] to consider trade-offs between "the degree of efficiency" of a DMU and its sensitivity to perturbations. We hope that this paper motivates research on such trade-offs.

\section{Acknowledgement}

This research was partially supported by Grant No. 067-0000000-1076 of the Ministry of Science, Education and Sports of the Republic of Croatia. We would like to acknowledge a suggestion from W. W. Cooper to the second author to examine the relationship between Tolerance sensitivity and sensitivity in DEA.

\section{References}

[1] Charnes A, Cooper W. W., Golany, L., Seiford, L. M. and Stutz, J. (1985). Foundations of data envelopment analysis for Pareto-Koopmans efficient empirical production functions. Journal of Econometrics, 30, 91-107. doi:10.1016/03044076(85)90133-2.

[2] Charnes A, Cooper W. W. and Rhodes E. (1978). Measuring the efficiency of Decision Making Units. European Journal of Operational Research, 2(6), 429-444. doi:10.1016/0377-2217(78)90138-8.

[3] Charnes, A., Haag, S., Jaska, P. and Semple, J. (1992). Sensitivity of efficiency calculations in the additive model of data envelopment analysis. Journal of Systems Sciences, 23(5), 789-798. doi:10.1080/00207729208949248.

[4] Charnes, A., and Neralić L. (1990). Sensitivity analysis of the additive model in data envelopment analysis. European Journal of Operational Research, 48, 332-341. doi:10.1016/0377-2217(90)90416-9.

[5] Charnes, A., Rousseau, J. J., and Semple, J. (1996). Sensitivity and stability of efficiency classifications in data envelopment analysis. The Journal of Productivity Analysis, 7(1), 5-18. doi:10.1007/bf00158473. 
[6] Cook, W. D. and Sieford, L. M. (2009). Data Envelopment Analysis (DEA) - Thirty years on. European Journal of Operational Research, 192(1), 1-17. doi:10.1016/j.ejor.2008.01.032.

[7] Cooper, W. W., Seiford, L. M. and Tone, K. (2007). Data Envelopment Analysis, $2^{\text {nd }}$ Edition. New York: Springer.

[8] Cooper, W. W., Li, S., Seiford, L. M., Tone, K., Thrall, R. M. and Zhu, J. (2001). Sensitivity and stability analysis in DEA: Some recent developments. Journal of Productivity Analysis, 15, 217-246.

[9] Emrouznejad A. (2013) Data Envelopment Analysis homepages. http: //www.DEAzone.com [Accessed on 28 July 2011].

[10] Filippi, C. (2005). A fresh view on the tolerance approach to sensitivity analysis in linear programming. European Journal of Operational Research, 167(1), 1-19. doi:10.1016/j.ejor.2004.01.050.

[11] Hladik, M. (2011). Tolerance analysis in linear systems and linear programming. Optimization Methods and Software. 26(3), 381-396. doi:10.1080/10556788.2011.556635.

[12] Jahanshahloo, G. R., Lotfi, F. H., Shoja, N., Tohidi, G. and Razavyan, S. (2005). A one-model approach to classification and sensitivity analysis in DEA. Applied Mathematics and Computation, 169(2), 887-896. doi:10.1016/j.amc.2004.09.068.

[13] Neralić, L. (1997). Sensitivity in Data Envelopment Analysis for arbitrary perturbations of data. Glasnik Matematicki, 32, 315-335.

[14] Neralić, L. and Wendell, R. E. (2004). Sensitivity in Data Envelopment Analysis using an approximate inverse matrix. Journal of the Operational Research Society, 55(11), 1187-1193. doi:10.1057/palgrave.jors.2601785.

[15] Schrijver, A. (1986). Theory of Linear and Integer Programming. New York: John Wiley \& Sons.

[16] Singh, S. (2010). Multiparametric sensitivity analysis of the additive model in Data Envelopment Analysis. International Transactions in Operational Research, 17(3), 365-380. doi:10.1111/j.1475-3995.2009.00735.x.

[17] Wang, H. F. and Huang, C. S. (1993). Multi-parametric analysis of the maximum tolerance in a linear programming problem. European Journal of Operational Research, 67(1), 75-87. doi:10.1016/0377-2217(93)90323-f.

[18] Ward, J. E. and Wendell, R. E. (1990). Approaches to sensitivity analysis in linear programming. Annals of Operations Research, 27(1), 3-38. doi:10.1007/bf02055188.

[19] Wendell, R. E. (1982). A preview of a tolerance approach to sensitivity analysis in linear programming. Discrete Mathematics, 38(1), 121-124. doi:10.1016/0012$365 \times(82) 90178-9$.

[20] Wendell, R. E. (1984). Using bounds on the data in linear programming: The tolerance approach to sensitivity analysis. Mathematical Programming, 29(3), 304322. doi:10.1007/bf02591999.

[21] Wendell, R. E. (1985). The tolerance approach to sensitivity analysis in linear programming. Management Science, 31(5), 564-578. doi:10.1287/mnsc.31.5.564.

[22] Wendell, R. E. (1997). Linear Programming 3: The Tolerance Approach. In: Gal, T. and Greenberg, H. J. (Eds.), Advances in Sensitivity Analysis and Parametric Programming (pp. 5-1-5-21). Boston/Dordrecht/London: Kluwer Academic Press.

[23] Wendell, R. E. (2004). Tolerance sensitivity and optimality bounds in linear programming. Management Science, 50(6), 797-803. doi:10.1287/mnsc.1030.0221. 
[24] Wendell, R. E. and Chen, W. (2010). Tolerance sensitivity analysis: Thirty years later. Croatian Operational Research Review, 1, 12-21. 